\title{
Study of Mechanical and Micro-Structural Effect on Alkaline Treated Sponge Gourd (Luffa aegyptiaca) Fibre Epoxy Composite
}

\author{
S. I. Ichetaonye ${ }^{1 *}$, D. N. Ichetaonye ${ }^{1}$, O. G. Tenebe ${ }^{2}$ \\ ${ }^{1}$ Department of Polymer and Textile Technology, Yaba College of Technology, Yaba, Lagos \\ ${ }^{2}$ Department of Polymer Technology, Nigerian Institute of Leather \& Science Technology, Zaria, Nigeria \\ Email: "ik4simon@yahoo.com
}

Received 17 November 2015; accepted 1 February 2016; published 4 February 2016

Copyright (C) 2016 by authors and Scientific Research Publishing Inc.

This work is licensed under the Creative Commons Attribution International License (CC BY). http://creativecommons.org/licenses/by/4.0/

c) (i) Open Access

\begin{abstract}
In this present work, the study of mechanical and micro-structural effect on alkaline treated sponge gourd fibre epoxy composite has been investigated experimentally. Composite laminates are fabricated by hand lay-up technique. Scanning Electro Microscope analysis on the composite materials is performed. A group of neat epoxy samples is fabricated for comparison purpose. Samples are analysed for their mechanical properties to establish an alkaline effect on sponge gourd. Indeed, a maximum value of strength and strain is observed over $20 \%$ filler loading for 24 hrs treated fibre composite.
\end{abstract}

\section{Keywords}

Sponge Gourd, Fibre, Flexural, Modulus

\section{Introduction}

During the last decade, natural fibre reinforced polymeric composites which substitute glass reinforcement, have witnessed considerable growth. This is attributed to their unique properties. These materials have the potential advantages of weight-saving (light material), lower raw material price from natural origin, and "thermal recycling" or the ecological advantages of using resources which are renewable. Due to the importance of natural fibre reinforced composite many non-structural components for the automotive and other sectors are now made from natural fibre composite materials [1]. These materials are largely based on polypropylene, polyester and polyamide matrices incorporating natural fibres, such as sponge gourd (Figure 1), hemp etc. Another major area

\footnotetext{
${ }^{*}$ Corresponding author.
} 


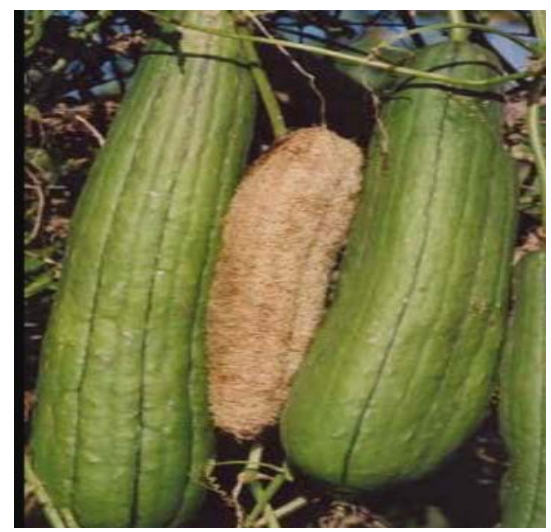

Figure 1. Dried sponge gourd.

that can extend the application of natural-fibre composites is the improvement of long-term performance including improved resistance to ultraviolet radiation and creep. Overall, natural-fibre composites are seen as potential materials for many engineering applications. However, there are still important issues that limit their future use, including long-term performance and the ability to be able to predict performance during service. Saheb et al. report that fracture mechanics can give great insight into the physical effects occurring within these composites which enable the production of natural-fibre composites with improved properties [2]. Debiprasad et al. mention that jute fibre composites enjoy excellent potential as wood substitutes in view of their low cost, easy availability saving in energy and commercialization [3]. Nor Azwin bt Ahad et al. mention that natural fibre offers an alternative to the technical reinforcing fibre because of their low density, good mechanical performance, ultimate availability and disposability [4].

The sponge gourd fruit is a cylindrical, smooth and dehiscent capsule, $20-50 \mathrm{~cm}$ long by 6 - $10 \mathrm{~cm}$ broad, which has a characteristic fibrous mesocarp. However, the seeds are numerous, dull black, elliptic-ovoid 10 - 12 $\mathrm{mm}$ long by $6-8 \mathrm{~mm}$ broad. It is best grown with a trellis support, requires lots of heat and lots of water to thrive [5] [6]. Gourds can be expected to mature around four months after planting after which it turns fibrous [7] [8]. Being a natural fibre it has recently attracted the attention of scientists and technologists because of the advantages that these fibres provide over conventional reinforcement materials and the development of natural fibre composites has been a subject of interest for the past few years. These natural fibres are low-cost fibres with low density and high specific properties. These are biodegradable and nonabrasive, unlike other reinforcing fibres. Also, they are readily available and their specific properties are comparable to those of other fibres used [9] $[10]$.

\section{Experimental}

\subsection{Materials}

1) Sponge Gourd

2) Epoxy (LY 556) and Hardener (HY 951)

3) Sodium Hydroxide

4) Distilled water

5) 88 Universal Mold Release Wax

6) Acetic Acid

\subsection{Equipment}

The equipment use was:

1) Digital weighing balance (Pocket Scale, Black AWS-100 g)

2) Steel mould (dumb-bell and sheet shape)

3) mm sieve, (YS-C-638)

4) Beaker and measuring cylinder (200 and 100 mils respectively) 


\subsection{Material Preparation}

Raw sponge gourd fibre was cut opened lengthwise, the dried seeds shaken out and the dried fibrous sun-dried for six (6) hours. It was later cut into smaller sizes, grounded and then sieved with $1.0 \mathrm{~mm}$ sieve to obtain fine fibre particles (Figure 2). Composite samples were fabricated with 5\%, 10\%, 15\%, 20\% and 25\% fillers in the ratio of 2:1 as in volume fraction using dumb-bell mould of $120 \mathrm{~mm} \times 30 \mathrm{~mm} \times 3 \mathrm{~mm}$ (Figure 3) and rectangular sheet mould of $187 \mathrm{~mm} \times 125 \mathrm{~mm} \times 3 \mathrm{~mm}$ (Figure 4) by hand laying method and finally left to air-cure for

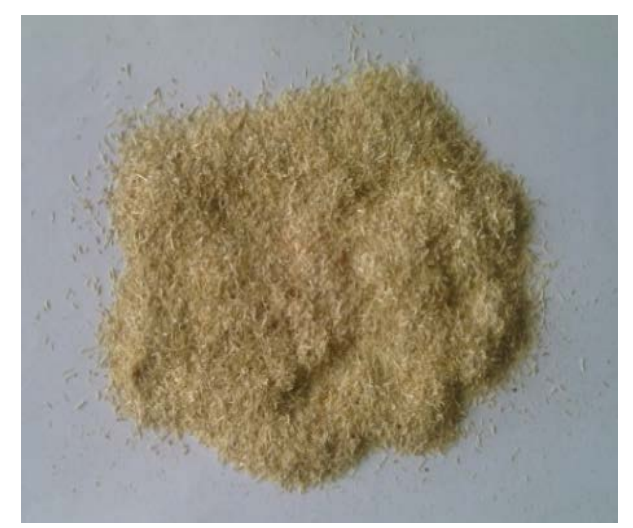

Figure 2. Grinded sponge fibres of particle size $1.0 \mathrm{~mm}$.

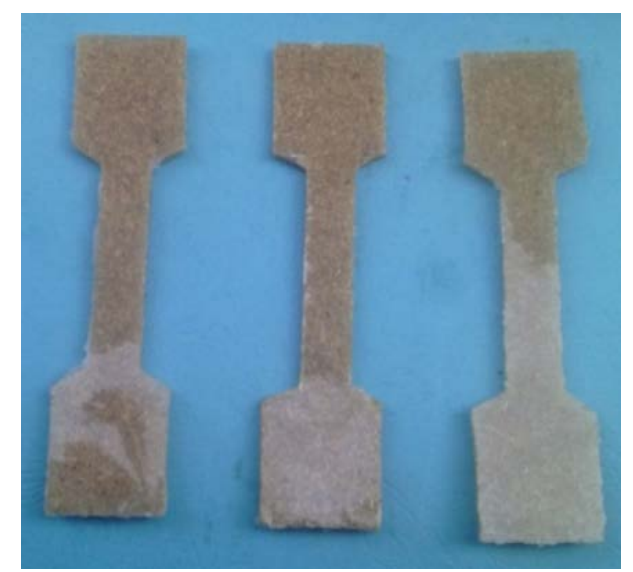

Figure 3. Dumb bell sponge gourd composite.

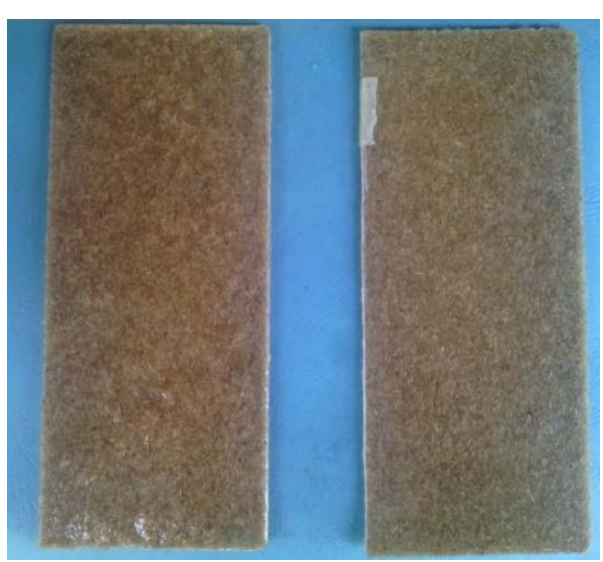

Figure 4. Rectangular sheet sponge gourd. 
24 hrs. Thereafter, the laminates are taken carefully without any damage. Specimens are cut for testing as per ASTM standards.

\subsection{Fibre Chemical Treatment}

The fibres were immersed in $\mathrm{NaOH}$ solution with a concentration of $20 \%$ for 12 and 24 hours respectively at room temperature. After treatment, the fibres were washed with $2 \%$ acetic acid and again washed under running water then finally allowed to dry at room temperature for 2 days.

\section{Characterization of Composite Materials}

\subsection{Determination of Swelling Behaviour}

The specimens were weighed dried $\left(\mathrm{W}_{\mathrm{i}}\right)$, immersed in $100 \mathrm{ml}$ volume of methanol solvent at room temperature for 48 hrs. After this, the samples were filtered and the excess solvent was removed patted dry with a lint free cloth and then the final weight $\left(\mathrm{W}_{\mathrm{f}}\right)$ was noted. The percent swelling was calculated using Equation (1).

$$
\text { Percent Swelling, }\left(\mathrm{P}_{\mathrm{s}}\right)=\frac{\left(\mathrm{W}_{\mathrm{f}}-\mathrm{W}_{\mathrm{i}}\right)}{\mathrm{W}_{\mathrm{i}}} \times 100
$$

\subsection{Determination of Tensile Strength}

The tensile strength test was conducted on a computerized universal testing machine. The tensile test was conducted in accordance with ASTM D 3039 method. The sample of $120 \mathrm{~mm}$ length was clamped into the two jaws of the machine. Each end of the jaws covered $30 \mathrm{~mm}$ of the sample. Reading of the tensile strength test instrument for Newton force and extension were initially set at zero (Figure 5). Tensile stress was applied until the failure of the sample and load-extension curve was obtained. Four (4) specimens of each sample have been used for the measurement of the above mechanical properties at ambient laboratory environment and average results are reported. From the obtained load and elongation values, the tensile strength and modulus are calculated.

\subsection{Determination of Flexural Strength Test}

Flexural strength of samples was also tested on the computerized universal testing machine. The three-point bend flexural test was conducted in accordance with ASTM D 790 method. The five (5) samples with desired dimensions and velocity of $0.2 \mathrm{~mm} / \mathrm{second}$ were tested (Figure 6). The $\sigma$ bh flexural strength, namely the maximum stress at break, was calculated using the formula

$$
\sigma \mathrm{bh}=\frac{3 \mathrm{FL}}{2 \mathrm{bh}^{2}}
$$

where, $\sigma \mathrm{bh}=$ Flexural strength; $\mathrm{b}=$ Width of specimen $(\mathrm{mm}) ; \mathrm{F}=$ Breaking force (Newton); $\mathrm{h}=$ Thickness of Specimen (mm); $\mathrm{L}=$ Support distance (mm).

\section{Results and Discussion}

\subsection{Swelling Behaviour}

Figure 7 is a graph of swelling behaviour against fibre content of sponge gourd fibre composite. The figure revealed that the swelling behaviour decreases as fibre content increases for all polymer composite having the least swelling behaviour percentage rate at $25 \%$ to be $7.06 \%, 4.87 \%$ and $4.23 \%$ for untreated, treated $12 \mathrm{hrs}$ and treated 24 hrs respectively compared to that of the control sample which has $18.18 \%$. Still on $25 \%$ fibre content, untreated polymer composite exhibits the highest swelling rate due to its greater affinity of water for $\mathrm{OH}$ groups present in the fibre reinforced polymer composites as well as the micro-cracks and non-uniform fibre-matrix adhesion compared to that of the treated polymer composite with very low affinity and better fibre matrix interaction.

\subsection{Ultimate Strength of Samples}

Figure 8 and Figure 9 show the strength variation with different percentage filler loading in tensile and flexural 


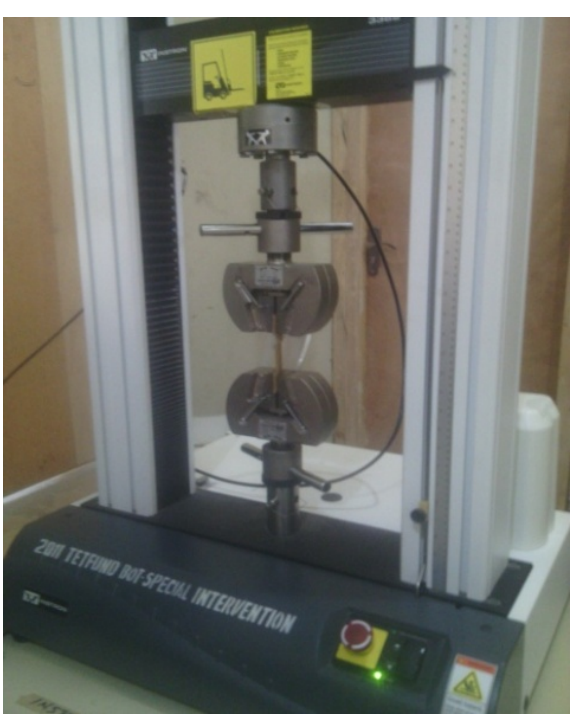

Figure 5. UTS machine sample loaded for tensile testing.

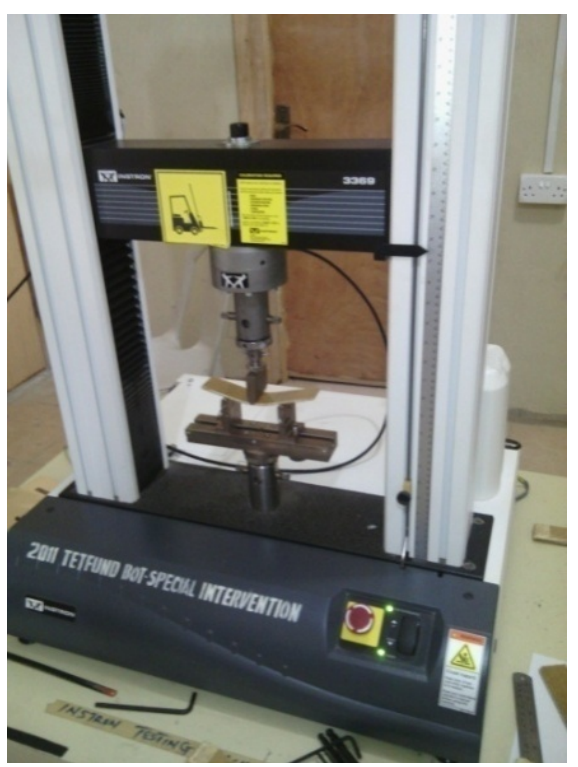

Figure 6. UTS machine sample loaded for flexural testing.

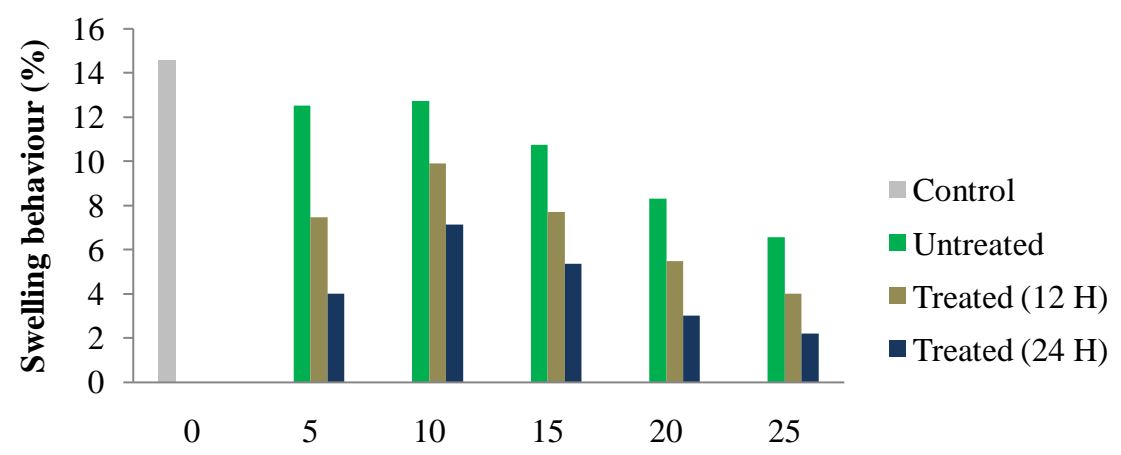

Filler loading (\%)

Figure 7. Swelling behaviour of sponge gourd fibre composite against filler loading. 


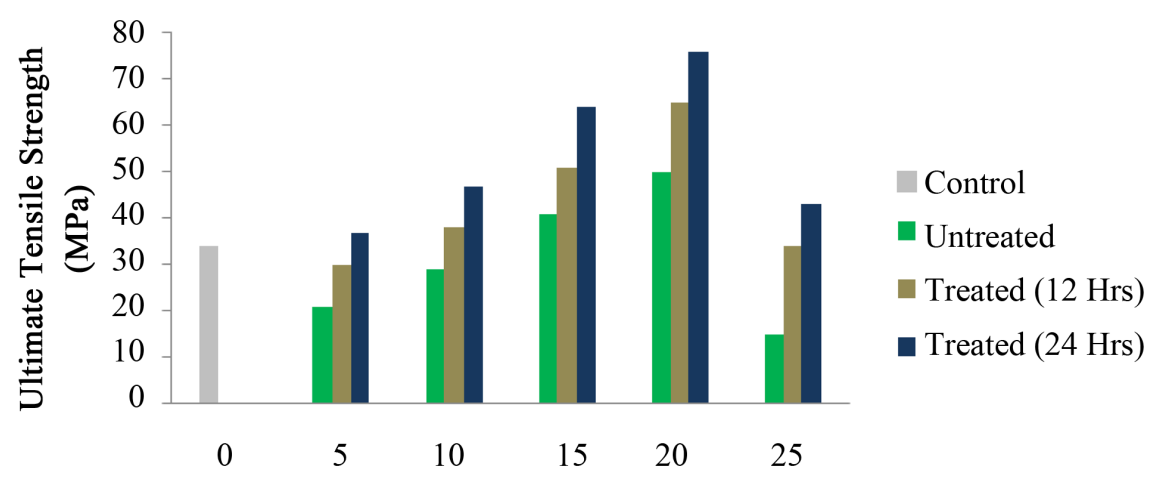

Filler Loading (\%)

Figure 8. Ultimate tensile strength of sponge gourd fibre composite against filler loading.

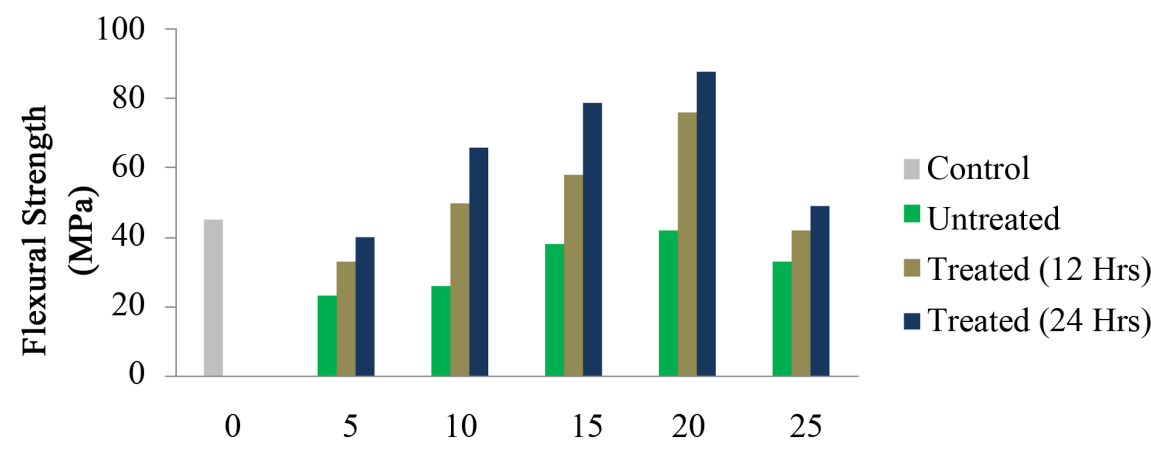

Filler Loading (\%)

Figure 9. Flexural strength of sponge gourd fibre composite against filler loading.

strength respectively. However, both figures indicate a gradual increase in both tensile and flexural strength up to $20 \%$ fibre content with $20 \%$ exhibiting the highest strength for treated polymer composite improved by the sponge gourd fibre than that of the control. Furthermore, at 25\% filler loading there was a decrease in strength for both tensile and flexural strength for treated and untreated polymer composite because maximum strength had been attained and further addition of fibre content disrupts the fibre-matrix adhesion due to insufficient wetting of the fibre with the matrix.

\subsection{Load at Break of Samples}

Figure 10 and Figure 11 show the effect of filler loading on both load at break and tensile modulus respectively. It can be seen that the properties of both untreated and treated (12 hrs and $24 \mathrm{hrs)} \mathrm{sponge} \mathrm{gourd} \mathrm{fibre} \mathrm{composite}$ increase gradually with increasing filler loading up to $20 \%$. This was due to the incorporation of sponge gourd fibres into the polymer matrix which enhanced the stiffness of the composites especially for 24 hrs treated fibres and gives better interaction between fibre and matrix compared to the control sample. However, 25\% filler loading exhibited a decrease in both properties due to poor wetting of fibre. The untreated fibre composite shows lower properties than the treated samples due to higher lignin content on its surface which tends to agglomerate through the formation of hydrogen bonding [11].

\subsection{Micro-Structural Analysis}

Figures 12-14 show the phase morphology of sponge gourd fibre composite with untreated and treated (12 hrs and 24 hrs) filler loading of 5\%. From the micrographs, it is clearly evident that the 24 hrs treated composite gives better interfacial interaction than the untreated and treated $12 \mathrm{hrs}$ composite. 


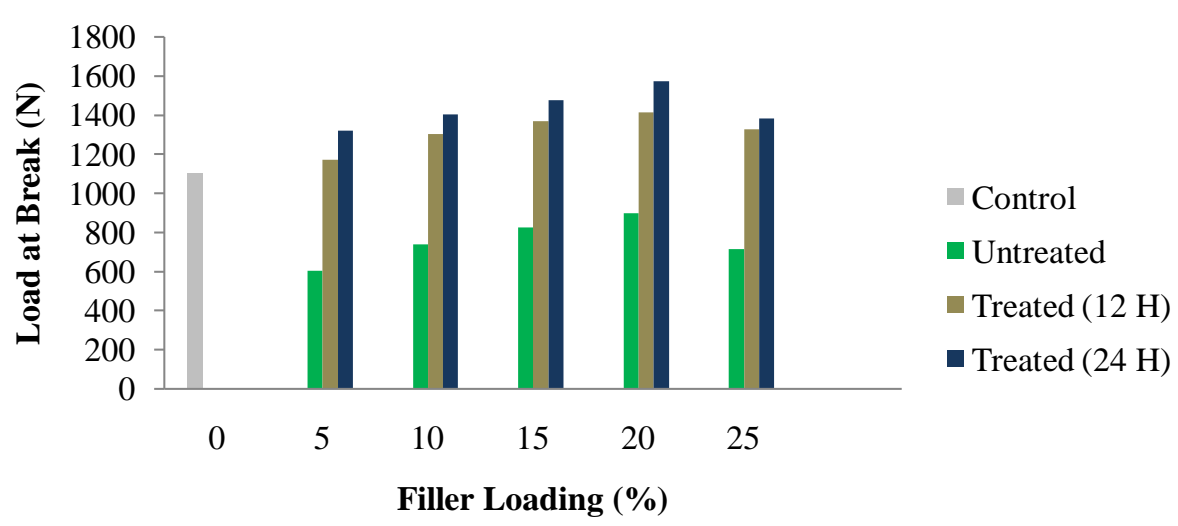

Figure 10. Load at break of sponge gourd fibre composite against filler loading.

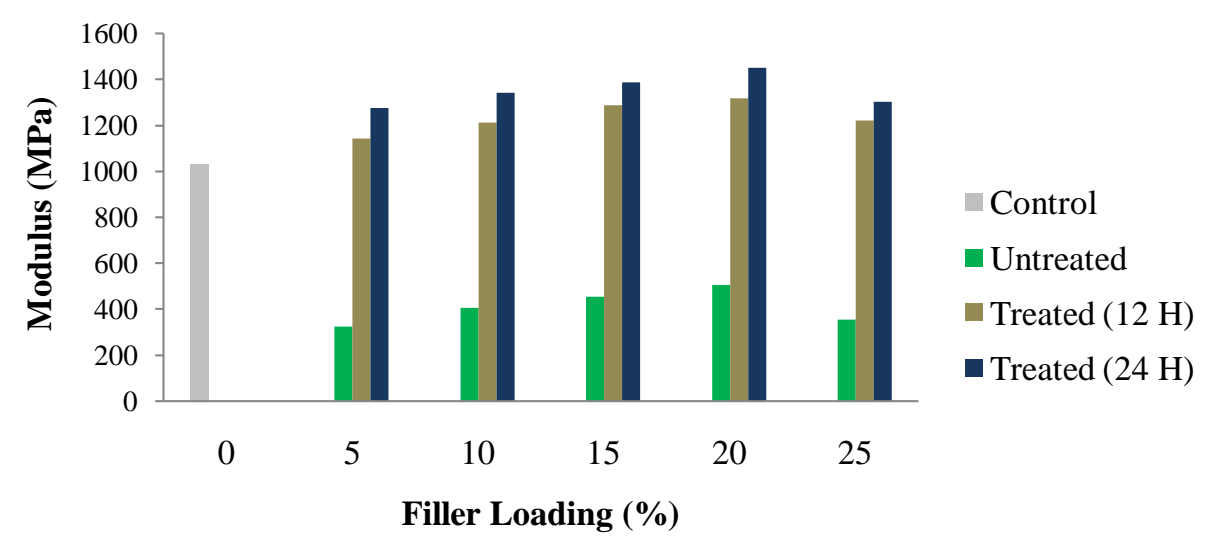

Figure 11. Modulus of sponge gourd fibre composite against filler loading.

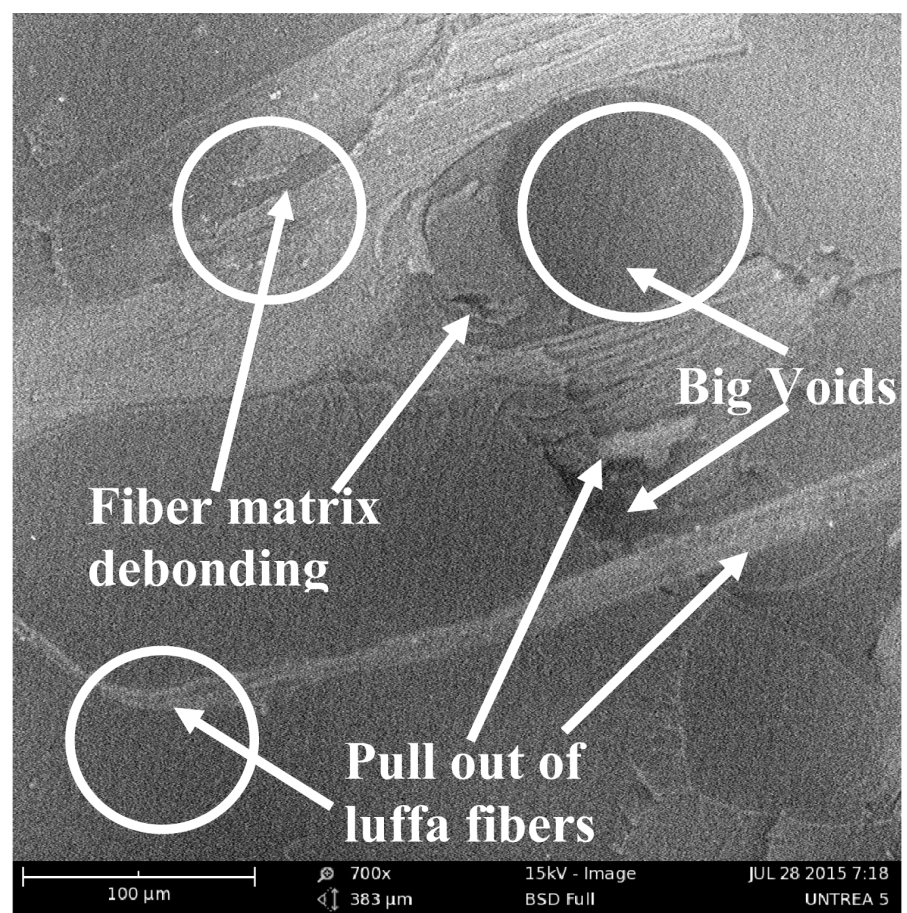

Figure 12. SEM image of sponge gourd fibre composite at filler loading of $5 \%$ untreated. 


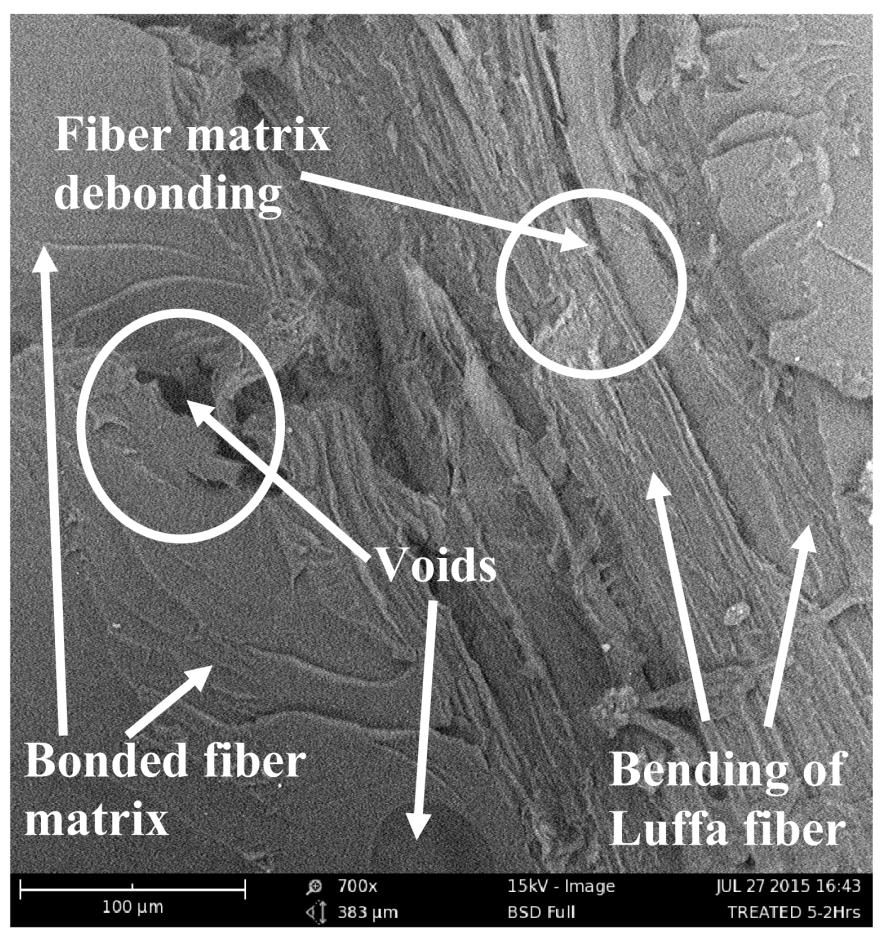

Figure 13. SEM image of sponge gourd fibre composite at filler loading of $5 \%$ treated for $12 \mathrm{hrs}$.

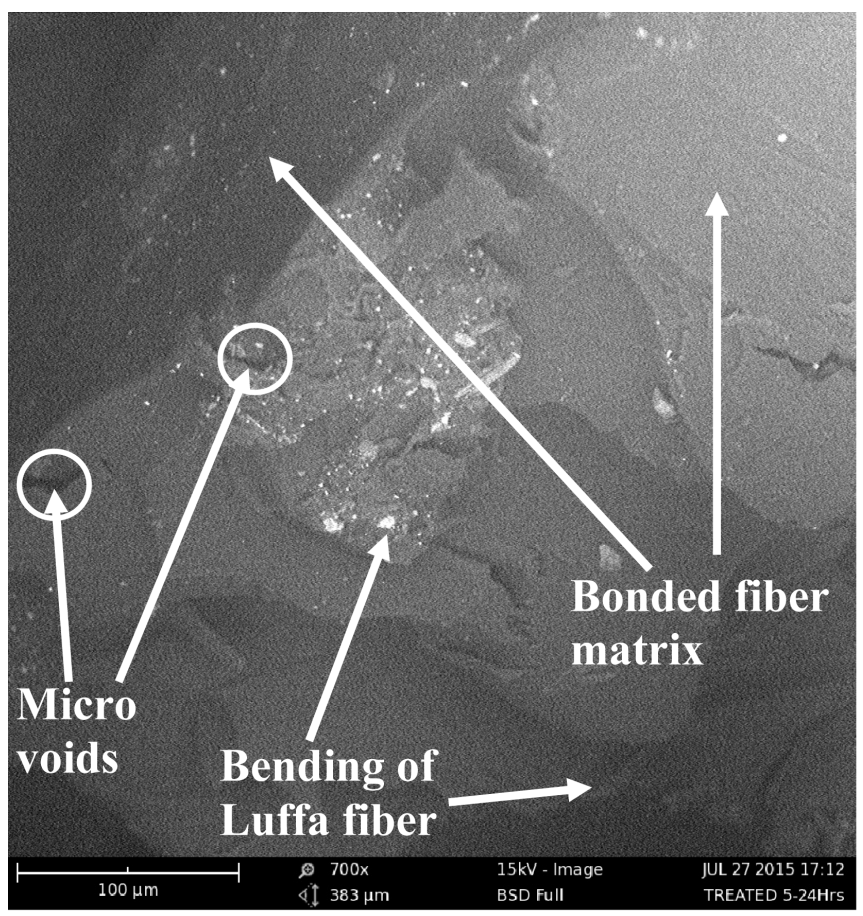

Figure 14. SEM image of sponge gourd fibre composite at filler loading of $5 \%$ treated for 24 hrs.

\section{Conclusion}

The study of mechanical and micro-structural effect of alkaline treated sponge gourd (luffa ageyptiaca) fibre 
epoxy composite is examined as a function of fibre loading. From the results generated, it can be established that $\mathrm{NaOH}$ pre-treatment of sponge gourd fibre has better reinforcing properties than the untreated fibres. The treatment is observed to have improved the flexural and tensile properties i.e. tensile strength, load at break and modulus of the composite as well as decreasing the swelling behaviour of the composite. However, $20 \%$ filler loading for 24 hrs treated fibre composite gives the best reinforcing/mechanical properties compared to other composites due to the adhesion between sponge gourd fibre and the polymer matrix.

\section{References}

[1] Jyoti, P.D. and Mishra, S.C. (2012) Processing and Properties of Natural Fibre-Reinforced Polymer Composite. Journal of Materials, 10, 27-33.

[2] Saheb, D.N. and Jog, J.P. (1999) Natural Fibre Polymer Composite: A Review, Advances in Polymer Technology. Journal of Polymers and the Environment, 18, 351-363.

[3] Debiprasad, G., Kousik, D., Palash, P. and Subhankar, M. (2012) Jute Composites as Wood Substitute. International Journal of Textile Science, 6, 84-93.

[4] Nor, A.B.A., Noraziana, P., Noraziana, M., Siti, S.I., Khairul, N. and Ying, M.H. (2009) Effect of Chemical Treatment on the Surface of Natural Fibre. Journal of Nuclear and Related Technologies, 6, 155-158.

[5] Davis, J.M. (1996) Luffa Sponge Gourd Production Practices for Temperate Climates. Horticulture Science, 29, 263266.

[6] De Courley, C. (1998) Luffa Research. A Wrap-Up Report. Small Farm Today, 4, 19-22.

[7] Davis, J.M. (1996) Luffa Sponge Gourd Production Practices for Temperate Climates. Horticulture Science, 29, 263266.

[8] Myrtha, K., Holia, O. and Anung, S. (2007) Physical and Mechanical Properties of Natural Fibres Filled Polypropylene Composites and Its Recycle. Polymer Engineering Science, 7, 393-396.

[9] Mallick, P.K. (2007) Fibre Reinforced Composites. CRC Press, London, New York, 1-15.

[10] Huo, S., Fuqua, M., Chevali, V. and Ulven, C. (2010) Effects of Natural Fibre Surface Treatments and Matrix Modification on Mechanical Properties of Their Composites. SAE Technical Paper, 01, 35-45.

[11] Onyeagoro, G.N. and Enyiegbulam, M.E. (2012) Physico-Mechanical Properties of Cellulose Acetate Butyrate/Yellow Poplar Wood Fibre Composites as a Function of Fibre Aspect Ratio, Fibre Loading, and Fibre Acetylation. International Journal of Basic and Applied Science, 1, 385-397. 